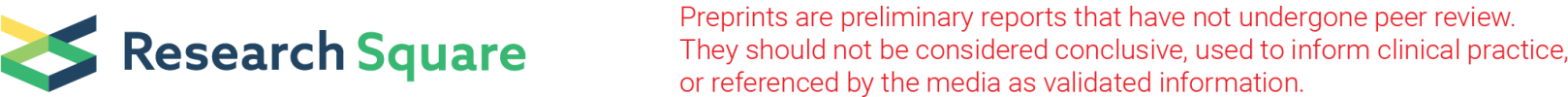

\section{Could the disclosure of individual health results hamper biobanking? Findings from a survey on potential donors screened for depression}

Virgilia Toccaceli ( $\sim$ virgilia.toccaceli@iss.it )

Scuola Universitaria Professionale della Svizzeria Italiana Dipartimento economia aziendale sanita e sociale

Corrado Fagnani

Istituto Superiore Di Sanita

Antonella Gigantesco

Istituto Superiore Di Sanita

Picardi Angelo

Istituto Superiore Di Sanita

Maria Antonietta Stazi

Istituto Superiore Di Sanita

Research article

Keywords: Research Biobanks, donors, individual health results, depression, mental health

Posted Date: December 5th, 2019

DOI: https://doi.org/10.21203/rs.2.18268/v1

License: (c) (1) This work is licensed under a Creative Commons Attribution 4.0 International License.

Read Full License 


\section{Abstract}

Background: Research based on the use of biological material stored in biobanks together with health information and clinical data may produce new strategies for prevention, early detection, accurate diagnoses, and tailored treatments. A postal survey on the knowledge and attitude towards research biobanking was conducted among a sample of individuals previously recruited in urban primary care services in Rome (Italy), and screened for major depressive disorder (MDD) (SET-DEP project). The present study aims at exploring the attitude of these participants towards the potential future disclosure of individual health results when participating in research biobanking.

Methods: The survey was conducted with a validated questionnaire on the knowledge and preferences about donation for research biobanks; self-perceived health was collected and The Satisfaction With Life Scale was administered. Means and percentages were compared across groups by using t test or ANOVA and chi-square test respectively.

Results: Out of 416 subjects who underwent the screening for MDD, 51 (12.2\%) subjects responded to the mail questionnaire. The large majority of respondents (42 subjects, $>82 \%$ ) declared they would agree to the potential use of their biological samples for biomedical research purposes only if no feedback results regarding health or predisposition to diseases would be given to them. The agreement towards research biobanking was not associated with satisfaction with life (ANOVA: $p=0.82$ ) or self-perceived health (chisquare test: $p=0.98)$.

Conclusions: It is widely recognized that willingness to donate diminishes when the research scenario raises moral, religious or political concerns. Nevertheless, other emerging concerns, more deeply linked to an individual psychosocial sphere, may also affect research biobanking. Our findings show a prevailing preference opposing the disclosure of individual health results not only among MDD positive screened but also - and even more - among MDD negative screened participants. This suggests that there might be relevant personal factors influencing people's preferences with respect to the feedback of individual results in the case of psychiatric disorders such as depression. Moreover, these preferences do not seem to depend on the perceived heath status or subjective wellbeing of respondents.

\section{Introduction}

The importance and value of biobanking in health research has been growing during the last decades mostly due to genetic knowledge advancements and technology innovation. In the context of psychiatric disorders, research based on the use of biological material stored in biobanks together with health information and clinical data may produce new strategies for prevention, early detection, accurate diagnoses, and tailored treatments (1). Among psychiatric disorders, major depressive disorder (MDD) is the most frequent, persistent and debilitating disorder occurring in the general population, with an estimated lifetime prevalence of approximately $12.8 \%$ in Europe (2). Moreover, MDD has major public health implications. In fact, it is the fourth leading cause of worldwide disease burden accounting for $12 \%$ 
of all years lived with disability, and has severe consequences in terms of economic costs (3). In a high proportion of patients, neither is MDD recognized nor treated adequately (4).

Despite what has been addressed as a "moral duty" to participate in research biobanks (5), it is important to highlight the emerging and somewhat unexpected importance of psycho-social wellbeing of potential donors for research biobanks. It is, in fact, one of the core welfare interests at stake in the process of establishing an organized collection of biological samples and associated data for health research. Coping with psycho-social wellbeing represents a central ethical duty that the biobanking endeavour has to accomplish. In some respects, this "core" interest enlarges the concept of individual's welfare in research on which the ethical debate has been focusing for a long time (6), placing this kind of interests, in our opinion, in the highest ranking of the ethical issues to be addressed by both researchers and IRBs.

The present study is part of the SET-DEP project (Screening and Enhanced Treatment for DEPression in Primary care, in Rome, Italy. Project funded by the Italian Ministry of Health in 2008) (7), whose main objective was to test the feasibility and effectiveness of a program for early detection and treatment of depression in primary care. One secondary objective of the SET-DEP project was the donation and biobanking of saliva from the participants for future research purposes. The study describes specific results obtained with a mail survey that explored, beforehand, the attitude of the SET-DEP participants towards biological material donation for research purposes. In particular, the focus was on the importance these potential donors attributed to the future disclosure of individual health results.

The Ethics Board of the Istituto Superiore di Sanità approved the SET- DEP project on June 17th, 2008 and an informed consent procedure was adopted to conduct this cross-sectional survey.

\section{Materials And Methods}

The present study aims at exploring the attitude of the SET-DEP participants towards the potential future disclosure of individual health results when participating in research biobanking, with the hypothesis that the agreement towards donation for research purposes might be influenced by perceived health and/or by satisfaction with life.

\section{Study population}

Participants were healthy individuals recruited from 13 urban general internal medicine practices, located in central Rome, Italy, from January 2009 to June 2010. They were previously asked to undergo a screening for MDD by means of specific questionnaires. The study inclusion criteria were age 18-65 and absence of psychosis or severe cognitive impairment as clinically determined by the primary care physicians according to anamnesis, prescription medicines, and medical history. The physicians or their assistants informed the eligible participants about the SET-DEP project, its aims and objectives including a future collection of saliva for research purposes; fliers describing the project were available in the waiting room. 
After the screening for MDD, at the same time of the follow-up interviews or shortly after, a survey was launched. Both MDD positive and negative SET-DEP participants were administered a postal questionnaire to investigate preferences and concerns about biological material donation for research purposes.

\section{Measures and questionnaires:}

The survey was conducted with a form for the collection of socio-demographic information, and with a validated questionnaire on the knowledge, attitude and willingness to donate biological material for research purposes used in previous research by our group (8). In particular, the item considered in the present study explored the agreement of respondents on the potential use of their biological samples for research purposes. Participants acceptance of disclosure was operationalized as different levels of agreement with the following statement: "Would you agree with the potential use of your biological samples for research purposes?" Response statements were: a) "Yes, I would definitely agree"; b) "Yes, but only if results regarding my health or predisposition to diseases will be given to me"; c) "Yes, but only if results regarding my health or predisposition to diseases will not be given to me"; d) "No, I would not agree"; e) "I don't know". Furthermore, self-perceived health was measured on a four-point Likert scale (categories: "Bad", "Neither Bad nor Good", "Good", "Excellent") and the well-known and widely validated "Satisfaction With Life Scale" (SWLS) (9) was administered. Life satisfaction is a subjective, cognitive evaluation of an individual's life as a whole based on the fit between personal goals and achievements (10). It is an indicator of subjective well-being, one of the main dimensions of mental health.

\section{Statistical analyses}

Descriptive analysis was performed by reporting means (with standard deviations) for continuous variables and percentages for categorical variables. To explore the association between variables, means and percentages were compared across groups by using t test or ANOVA and chi-square test respectively, with alpha set at $5 \%$.

\section{Results}

Four hundred and two individuals out of the 416 ones who underwent the screening for MDD received the mail questionnaire, and 51 subjects responded (12.2\%). Participants to this study showed a similar distribution by sex, age, marital status and education level compared to the reference SET-DEP cohort, with only a slight over-representation of subjects with a university degree in our sample. The 51 respondents (12 males, 39 females) represented ages 19 to 66 years (median age 51 years). More than half were married and about $90 \%$ of them had at least a high school diploma. Of all respondents, 21 (41\%) screened positive for MDD (similar positivity rate compared to the reference SET-DEP cohort, 37\%), with no difference in the positivity rate between genders [5 out of 12 males $(41.67 \%)$ screened positive, 16 out of 39 females (41.03\%) screened positive; chi-square test: $p=0.97]$. Positive subjects tended to be older (mean age 53 years) than negative ones (mean age 46 years; t test: $p=0.06$ ) (Table 1). 
Table 1

Characteristics of the study sample

\begin{tabular}{|lll|}
\hline Variables & N (\%) & Mean (SD) \\
\hline Age (years) & & $48.7(12.3)$ \\
\hline Gender & $12(23.5)$ & \\
\hline Male & $39(76.5)$ & \\
\hline Female & & \\
\hline Education & $0(0)$ \\
\hline Primary school & $2(3.9)$ & \\
\hline Secondary school & $3(5.9)$ & \\
\hline Vocational school & $24(47.1)$ & \\
\hline High school & $2(3.9)$ & \\
\hline 3-year degree & $20(39.2)$ & \\
\hline 5-year degree & & \\
\hline Marital status & $14(27.4)$ & Mean age (SD) \\
\hline Single & $30(58.8)$ & \\
\hline Married or living with a partner & $6(11.8)$ \\
\hline Separated, divorced or widowed & $1(2.0)$ \\
\hline Missing & $30(58.8)$ & $46.1^{*}(13.6)$ \\
\hline Screening for depression & & \\
\hline Positive & & \\
\hline Negative & & \\
\hline * $p=0.06$ (t test) & & \\
\hline
\end{tabular}

As expected, MDD negative subjects reported a better self-perceived health (chi-square test: $p=0.013$ ) and a higher level of life satisfaction ( $t$ test: $p<0.001)$ compared to positive ones (Table 2 ).

Seven respondents (14\%) declared they had already donated biological material (blood, saliva, urine, or other tissues) for research purposes in other research settings. Noteworthy, the large majority of respondents (42 subjects, $>82 \%$ ) declared they would agree to the potential collection and use of their biological samples for biomedical research purposes only if no feedback results regarding health or predisposition to any diseases would be given to them. This proportion was even higher in negative 
compared to positive subjects at the screening for MDD ( $90 \%$ vs $71 \%$ respectively; chi-square test: $p=$ 0.025). The percentages of other response categories were negligible [“No, I do not agree" (5.9\%), "Yes, but only if results regarding my health or predisposition to diseases will be given to me" (3.9\%), "Yes, I definitely agree" (2\%), "I don't know" (5.9\%)]. Finally, the agreement towards research biobanking was not associated with self-perceived health (chi-square test: $p=0.98$ ) or life satisfaction (ANOVA: $p=0.82$ ). 
Table 2

Description of survey items by MDD screening test results

\begin{tabular}{|c|c|c|c|c|c|c|}
\hline \multirow[t]{2}{*}{ Variables } & \multicolumn{2}{|c|}{ MDD Negative } & \multicolumn{2}{|c|}{ MDD Positive } & \multicolumn{2}{|l|}{ Total } \\
\hline & $\mathrm{N}(\%)$ & $\begin{array}{l}\text { Mean } \\
\text { (SD) }\end{array}$ & $\mathrm{N}(\%)$ & $\begin{array}{l}\text { Mean } \\
(\mathrm{SD})\end{array}$ & $\mathrm{N}(\%)$ & $\begin{array}{l}\text { Mean } \\
\text { (SD) }\end{array}$ \\
\hline \multicolumn{7}{|l|}{$\begin{array}{l}\text { Agreement on the use of a biological } \\
\text { sample for research purposes }{ }^{\#}\end{array}$} \\
\hline I don't agree & $0(0 \%)$ & & $\begin{array}{l}3 \\
(14.3 \%)\end{array}$ & & $\begin{array}{l}3 \\
(5.9 \%)\end{array}$ & \\
\hline Only if no results will be given to me & $\begin{array}{l}27 \\
(90 \%)\end{array}$ & & $\begin{array}{l}15 \\
(71.4 \%)\end{array}$ & & $\begin{array}{l}42 \\
(82.4 \%)\end{array}$ & \\
\hline Only if results will be given to me & $\begin{array}{l}2 \\
(6.7 \%)\end{array}$ & & $0(0 \%)$ & & $\begin{array}{l}2 \\
(3.9 \%)\end{array}$ & \\
\hline I definitely agree & $\begin{array}{l}1 \\
(3.3 \%)\end{array}$ & & $0(0 \%)$ & & $1(2 \%)$ & \\
\hline I don't know & $0(0 \%)$ & & $\begin{array}{l}3 \\
(14.3 \%)\end{array}$ & & $\begin{array}{l}3 \\
(5.9 \%)\end{array}$ & \\
\hline \multicolumn{7}{|c|}{$\begin{array}{l}\text { Previous donation of a biological sample } \\
\text { for research purposes }\end{array}$} \\
\hline No & $\begin{array}{l}27 \\
(90 \%)\end{array}$ & & $\begin{array}{l}15 \\
(71.4 \%)\end{array}$ & & $\begin{array}{l}42 \\
(82.4 \%)\end{array}$ & \\
\hline Yes & $3(10 \%)$ & & $\begin{array}{l}4 \\
(19.1 \%)\end{array}$ & & $\begin{array}{l}7 \\
(13.7 \%)\end{array}$ & \\
\hline I don't know & $0(0 \%)$ & & $\begin{array}{l}2 \\
(9.5 \%)\end{array}$ & & $\begin{array}{l}2 \\
(3.9 \%)\end{array}$ & \\
\hline \multicolumn{7}{|l|}{ Self-perceived health } \\
\hline Bad & $\begin{array}{l}2 \\
(6.7 \%)\end{array}$ & & $\begin{array}{l}4 \\
(19.1 \%)\end{array}$ & & $\begin{array}{l}6 \\
(11.8 \%)\end{array}$ & \\
\hline Neither bad nor good & $\begin{array}{l}12 \\
(40 \%)\end{array}$ & & $\begin{array}{l}15 \\
(71.4 \%)\end{array}$ & & $\begin{array}{l}27 \\
(52.9 \%)\end{array}$ & \\
\hline Good & $\begin{array}{l}14 \\
(46.7 \%)\end{array}$ & & $\begin{array}{l}2 \\
(9.5 \%)\end{array}$ & & $\begin{array}{l}16 \\
(31.4 \%)\end{array}$ & \\
\hline Excellent & $\begin{array}{l}2 \\
(6.7 \%)\end{array}$ & * & $0(0 \%)$ & * & $\begin{array}{l}2 \\
(3.9 \%)\end{array}$ & \\
\hline SWL score & & $\begin{array}{l}25.6^{\wedge} \\
(4.4)\end{array}$ & & $\begin{array}{l}18.6^{\wedge} \\
(8.2)\end{array}$ & & $\begin{array}{l}22.7 \\
(7.1)\end{array}$ \\
\hline
\end{tabular}

\# For complete answer categories, see text; MDD, Major Depressive Disorder; SWL, Satisfaction with life. 
* $p=0.013$ (chi-square test)

$\wedge p=0.0003$ ( $t$ test)

\section{Discussion}

Some degree of lack of knowledge regarding study participants' perspectives and opinions on biobanks is well known among stakeholders such as scientists, researchers and biobanks' sponsors (11).

It is our opinion that, especially for conditions of high relevance in public health such as psychiatric illnesses, the gap of knowledge has to be promptly filled in order to favour biobanking activities, and most of all, individuals' aware participation to these activities.

So far, many authors drew great attention on the effect of "non-welfare" interests on willingness to donate to a biobank $(12,13,14)$. It is widely recognized, in fact, that the willingness diminishes when the research scenario raises moral, religious or political concerns. Nevertheless, it is worth considering that other emerging concerns, more deeply linked to an individual psychosocial sphere, may also affect the compliance.

In our study, we found that an extremely high percentage of subjects expressed concern about the potential disclosure of individual results, no matter whether these results were study results or incidental findings; they indirectly stated that such a disclosure would negatively affect their willingness to donate for research purposes. This finding is quite odd in the landscape of studies on the same topic. In fact, regardless of the settings and type of participants surveyed [e.g., general population $(15,16)$, general population vs. research participants $(17)$, patients $(18,19)$, biobank effective participants vs. potential participants $(20,21)]$, a great majority of studies found that the disclosure of individual results is well accepted and can be even positively influential in the decision to donate. However, a few studies have already highlighted certain specific signals of the difficulties in disclosing individual health information. In this trend, for instance, Janssen and colleagues reported that patients are interested in receiving information when the disclosure regards very low risk events (22). In the same line, Meulenkamp and colleagues (23) found that, even if only low percentages of individuals (both patients and healthy subjects) do not want to receive aggregate or individual results, the type of results in terms of severity of the conditions in question really matters for decision. Moreover, they also found that anxiousness, as perceived by respondents, is associated with lower preference for results information. The same effects of anxiousness are detectable in other studies (24). Among the SET-DEP respondents who participated in our survey, we found a prevailing preference opposing the disclosure of individual results, not only among those who screened positive for depression but also - and even more - among those who screened negative. This result suggests that there may be relevant personal factors influencing people's preferences with respect to the feedback of individual results when psychiatric disorders such as depression are at stake. Additionally, these preferences do not seem to depend on the health status or subjective wellbeing as perceived by respondents. We might formulate a first straightforward hypothesis that interest in knowing the results of biosample testing for this kind of disorders could be markedly 
affected by fears of negative consequences such as labelling, social stigma or discrimination (25) or risk to become more prone to depression and to compromise the successful management of symptoms. Considering that respondents voluntarily participated in the screening for MDD, a certain degree of concern for depression and mental disorders can be envisaged in all the respondents, irrespective of the results of the screening itself. It is of note that the results of the screening had already been communicated to the participants at the time the present survey started. Taking into account this last element, the hypothesis may be enriched by a further consideration about the strength of a generalized concern not only for ascertained mental illnesses but also for a threat of mental illnesses.

Further factors, such as family history or social and cultural features of the individuals might also contribute to explain these results. Obviously, a pure selection bias may have occurred; our study sample represents "healthy" subjects coming from the general population who voluntarily joined a screening programme, whose decision may have been highly influenced by fear of being at high risk for anxiety or depression on the basis, for example, of a specific personal or family history of the disease. Although this is possible, we observed, at the same time, that the preference opposing the disclosure of individual results is not driven by the perceived health status of respondents or by the level of subjective wellbeing that can be considered as a proximal measure of anxiety and depression. This last element deserves further and deeper investigations. Furthermore, our sample shows a high education level that in itself has often been associated with a major selectiveness regarding the type of "information" individuals wish to receive when participating in research (26).

\section{Limitations And Strengths}

The survey has two main methodological limitations. First, a low response rate led to a small sample size that makes the results prone to random variability; in this respect, we cannot ignore that the SET-DEP project may have strained the potential participants as the request to participate in this survey partly overlapped with other requests and the follow-up interviews planned in the project. A second limitation is that the questionnaire item regarding the disclosure of individual health results did not specify any "diseases" or "syndromes", and consequently, no information was provided to respondents about different therapeutic scenarios. Therefore, we cannot exclude that the knowledge of specific treatments or cures regarding the health results to disclose might produce different findings in a similar survey.

Strengths of our study are the use of a validated questionnaire and the fact that our sample, though small, closely reflects the main socio-demographic characteristics and the positivity rate of the reference SET-DEP cohort. As a whole, the rate of refusal of health information disclosure among these potential donors is too high to be neglected.

\section{Conclusions}

The study provides cues for reflection on the importance of welfare interests and, in particular, of psychosocial wellbeing of individuals and its potential impact on research that makes use of biological 
sample collections. Considering the small proportion of the SET-DEP study participants who replied to this survey, we may also be allowed to hypothesize that non-respondents were even less interested in biobank participation, and this could represent a real pitfall for research biobank enterprises.

Given the importance of biomedical research and biobanking activities in the field of mental disorders, we consider these preliminary results worth of further investigations in larger samples taking into account other explanatory factors not necessarily related to mental disorders. It will be of note to disentangle the complex interplay of personal, psychological and social factors shaping the contribution of study participants, as well as to assess the "burden" that healthy and diseased donors have to withstand to contribute to research biobanking.

\section{Declarations}

Acknowledgments: we are grateful to Mrs. Cristina D'Ippolito for the management of the Set-Dep Database.

Ethics approval and consent: the Ethics Board of the Istituto Superiore di Sanità, Rome (Italy) approved the SET- DEP project on June $17^{\text {th }}, 2008$ and an informed consent procedure was adopted to conduct this cross-sectional survey.

Consent for publication: Not applicable

Availability of data and materials: the minimum dataset analyzed during the current study is available from the Corresponding Author on reasonable request.

Funding: the study was part of the Project SET-DEP funded by the Ministry of Health in 2008

Competing interests: None

Authors' contributions: VT contributed to the study conception and design, supervised data collection and drafted the manuscript. CF contributed to the study design, performed statistical analysis and revised the manuscript for important intellectual content. AG contributed to the study conception and revised the manuscript for important intellectual content. AP contributed to the supervision of data collection and revised the manuscript for important intellectual content. MAS contributed to the study conception and revised the manuscript for important intellectual content.

\section{The authors have no conflict of interests to declare}

\section{References}

1. Roberts LW, Kim JP. Receptiveness to participation ingenetic research: A pilot study comparing views of people with depression, diabetes, or no illness. J Psychiatr Res. 2017;94:156-162. 
2. Alonso J, Angermeyer MC, Bernert S, et al. Investigators, European Study of the Epidemiology of Mental Disorders (ESEMeD) Project. Prevalence of mental disorders in Europe: results from the European Study of the Epidemiology of Mental Disorders (ESEMeD) project. Acta Psychiatr Scand Suppl. 2004;420:21-7.

3. Greenberg PE, Fournier AA, Sisitky T, et al. The economic burden of adults with major depressive disorder in the United States (2005 and 2010). J Clin Psychiatr. 2015;76:155-162.

4. Davidson JR, Meltzer-Brody SE. The underrecognition and undertreatment of depression: what is the breadth and depth of the problem? J Clin Psychiatr. 1999;60:4-9 discussion 10-11.

5. De Vries RG, Ryan KA, Gordon L, et al. Biobanks and the Moral Concerns of Donors: A Democratic Deliberation. Qual Health Res. 2018 Aug 10:1049732318791826. doi: 10.1177/1049732318791826. [Epub ahead of print] PubMed PMID: 30095038.

6. Heeney C, Kerr SM. Balancing the local and the universal in maintaining ethical access to a genomics biobank. BMC Med Ethics. 2017;18(1):80.

7. Picardi A, Lega I, Tarsitani L, et al. A randomised controlledtrial of the effectiveness of a program for early detection and treatment of depression in primary care. J Affect Disord. 2016;198:96-101.

8. Toccaceli V, Fagnani C, Gigantesco A, et al. Attitudes and willingness to donate biological samples for research among potential donors in the Italian Twin Register. J Empir Res Hum Res Ethics. 2014;9: 39-47.

9. Diener E, Emmons RA, Larsen RJ, et al. The satisfaction with life scale. J Pers Ass. 1985;49:71-75.

10. Pavot W, Diener E, Colvin CR, et al. Further validation of the Satisfaction with Life Scale: evidence for the cross-method convergence of well-being measures. J Pers Assess. 1991;57(1):149-61

11. Domaradzki J, Pawlikowski J. Public Attitudes toward Biobanking of Human Biological Material for Research Purposes: A Literature Review. Int J Environ Res Public Health. 2019;16(12). pii: E2209. doi: 10.3390/ijerph16122209. Review. PubMed PMID: 31234457; PubMed Central PMCID: PMC6617000.

12. Tomlinson T. Protection of non-welfare interests in the research uses of archived biological samples. In: Borry P, Dierickx K, editors. New Challenges for Biobanks: Ethics, Law, and Governance. Ant-werp, the Netherlands: Intersentia. 2009; 99-110.

13. De Vries RG, Tomlinson T, Kim HM, et al. The moral concerns of biobank donors: the effect of nonwelfare interests on willingness to donate. Life Sci Soc Policy. 2016;12:3.

14. Gornick MC, Ryan KA, Kim SY. Impact of non-welfare interests on willingness to donate to biobanks: an experimental survey. J Empir Res Hum Res Ethics. 2014;9:22-33.

15. Ahram $\mathrm{M}$, Othman $\mathrm{A}$, Shahrouri $\mathrm{M}$, et al. Factors influencing public participation in biobanking. Eur $\mathrm{J}$ Hum Genet 2014;22:445-51.

16. Fleming J, Critchley C, Otlowski M, et al. Attitudes of the general public towards the disclosure of individual research results and incidental findings from biobank genomic research in Australia. Intern Med J. 2015;45:1274-9. 
17. Yamamoto K, Hachiya T, Fukushima A, et al. A. Population-based biobank participants' preferences for receiving genetic test results. J Hum Genet. 2017;62:1037-48.

18. Breitkopf CR, Petersen GM, Wolf SM, et al. Preferences Regarding Return of Genomic Results to Relatives of Research Participants, Including after Participant Death: Empirical Results from a Cancer Biobank. J Law Med Ethics. 2015;43:464-75.

19. Haukkla A, Kujala E, Alha P, et al. The return of unexpected research results in a biobank study and referral to health care for heritable long QT syndrome. Pub Health Genom. 2013;16:241-50.

20. AllenNL, Karlson EW, Malspeis S, et al. Biobank participants' preferences for disclosure of genetic research results: perspectives from the OurGenes, OurHealth, OurCommunity project. Mayo Clin Proc. 2014;89:738-46.

21. Long CR, Stewart MK, Cunningham TV, et al. Health research participants' preferences for receiving research results. Clin Trials. 2016;13:582-91.

22. Janssen NB, Oort FJ, Fockens $P$ et al.Under what conditions do patients want to be informed about their risk of a complication? A vignette study. J Med Ethics. 2009;35(5):276-82. doi: 10.1136/jme.2008.025031. PubMed PMID: 19407030.

23. Meulenkamp TM, Gevers SK, Bovenberg JA, et al. Communication of biobanks' research results: what do (potential) participants want? Am J Med Genet A. 2010;152A(10):2482-92. doi: 10.1002/ajmg.a.33617. PubMed PMID: 20799322.

24. Partridge $A H$, Burstein $H J$, Gelman RS, et al. Do patients participating in clinical trials want to know study results? J Natl Cancer Inst. 2003;95(6):491-2. PubMed PMID: 12644548.

25. Newson AJ. Depression under stress: ethical issues in genetic testing. $\mathrm{Br} \mathrm{J}$ Psychiatry. 2009;195:189e90. $囚$

26. Flatau L, Reitt M, Duttge G, et al. Genomic information and a person's right not to know: A closer look at variations in hypothetical informational preferences in a German sample. PLoS One. 2018;20; 13:e0198249. 